\title{
BMJ Open mHealth tool to improve community health agent performance for child development: study protocol for a cluster-randomised controlled trial
} in Peru

Christopher Michael Westgard (D) , ${ }^{1,2}$ Natalia Rivadeneyra, ${ }^{2}$ Patricia Mechael $^{3}$

To cite: Westgard CM, Rivadeneyra N, Mechael P. mHealth tool to improve community health agent performance for child development: study protocol for a cluster-randomised controlled trial in Peru. BMJ Open 2019;9:e028361. doi:10.1136/ bmjopen-2018-028361

- Prepublication history for this paper is available online. To view these files, please visit the journal online (http://dx.doi. org/10.1136/bmjopen-2018028361).

Received 05 December 2018 Revised 25 September 2019 Accepted 26 September 2019

Check for updates

(c) Author(s) (or their employer(s)) 2019. Re-use permitted under CC BY-NC. No commercial re-use. See rights and permissions. Published by BMJ.

${ }^{1}$ Department of Maternal and Child Health, University of North Carolina at Chapel Hill Gillings School of Global Public Health, Chapel Hill, North Carolina, USA

${ }^{2}$ Elementos, Lima, Peru

${ }^{3}$ HealthEnabled, Cape Town,

South Africa

Correspondence to

Christopher Michael Westgard; cmwestgard@unc.edu

\section{ABSTRACT}

Introduction Cultivating child health and development creates long-term impact on the well-being of the individual and society. The Amazon of Peru has high levels of many risk factors that are associated with poor child development. The use of 'community health agents' (CHAs) has been shown to be a potential solution to improve child development outcomes. Additionally, mobile information and communication technology (ICT) can potentially increase the performance and impact of CHAs. However, there is a knowledge gap in how mobile ICT can be deployed to improve child development in low resource settings.

Methods and analysis The current study will evaluate the implementation and impact of a tablet-based application that intends to improve the performance of $\mathrm{CHAs}$, thus improving the child-rearing practices of caregivers and ultimately child health and development indicators.

The CHAs will use the app during their home visits to record child health indicators and present information, images and videos to teach key health messages. The impact will be evaluated through an experimental cluster randomised controlled trial. The clusters will be assigned to the intervention or control group based on a covariateconstrained randomisation method. The impact on child development scores, anaemia and chronic malnutrition will be assessed with an analysis of covariance. The secondary outcomes include knowledge of healthy child-rearing practices by caregivers, performance of CHAs and use of health services. The process evaluation will report on implementation outcomes. The study will be implemented in the Amazon region of Peru with children under 4. The results of the study will provide evidence on the potential of a mHealth tool to improve child health and development indicators in the region.

Ethics and dissemination The study received approval from National Hospital 'San Bartolome' Institutional Ethics Committee on 8 November 2018 (IRB Approval \#15463-18) and will be disseminated via peer-reviewed publications.

Trial registration number ISRCTN43591826.

\section{BACKGROUND}

In low-income and middle-low income countries, millions of children under the age of
Strengths and limitations of this study

- The intermediate variables will be assessed to measure the behaviour changes that occurs along the theory of change. The study will be able to evaluate the various mediator variables that are expected to contribute to the final objective.

- A process evaluation with qualitative assessments will be used to identify issues of implementation and inform the implementation plan for scalability.

- The mHealth tool and the monitoring and evaluation strategy provides a surveillance mechanism that will allow for continuous feedback on the use of the intervention tool and health status of the study group.

- Child development will be assessed through a parent reported survey which provides the potential for the parent to give inaccurate responses due to a social desirability bias. However, the instrument that was chosen for the assessment, the Caregiver-Reported Early Development Instrument, has shown to have a high correlation to results obtained from surveys that administer direct observation of the child.

- The secondary outcomes that measure changes of the CHAs will be underpowered due to limitations in the sample size of CHAs.

5 never reach their cognitive, linguistic and socioemotional potential. ${ }^{12}$ Chronic malnutrition, anaemia and iodine deficiency delay the physical and cognitive development of children, reduce their level of energy and weaken their immune system. Child development can also be delayed by low levels of early childhood stimulation. ${ }^{13}$ In the Peruvian Amazon, there is a high prevalence of various risk factors associated with developmental delay, especially in rural areas. A study conducted in 2017 showed that the prevalence of developmental delay in the Amazon of Peru was 26.7\%. In the Amazonian department of Loreto, the prevalence of childhood anaemia is $49.9 \%$ and chronic malnutrition is 
$23.6 \%$ (2017).$^{5}$ Those indicators are worse in indigenous communities. $^{6-9}$

The well-being of children in communities can be greatly increased by improving families' understanding of healthy child-rearing practices. Health-promotion strategies that establish an intercultural dialogue with families can do a better job at promoting healthy practices than promotion through the health centres. ${ }^{10}{ }^{11} \mathrm{~A}$ programme can train and support community members to conduct health promotion that can create behaviour changes and improve child development outcomes. Community health agents (CHAs) can play an essential role in reducing inequalities in health services by acting as a link between communities and the local health services. ${ }^{11-13}$ The CHA can establish a warm relationship with the caregiver, thus increasing the acceptance of health advice, improving the absorption of knowledge and getting caregivers involved in the identification and resolution of their own health problems. ${ }^{14-21}$ The positive impact of CHAs has been associated with a reduction in malnutrition, anaemia, malaria, cases of diarrhoea, improved child development and other health indicators. ${ }^{112223}$ Therefore, understanding and improving the performance of CHAs can greatly improve behavioural outcomes of caregivers, increase the utilisation of local health services and enhance the overall health and wellbeing of communities. ${ }^{14}$ 24-31

Several studies have shown that mobile information and communication technology (ICT) can improve the performance of CHAs in their ability to perform health promotion, collect and report timely information regarding family health, provide health services such as vaccines and refer families to appropriate local health services. $^{32-39}$ Additionally, when a mobile ICT tool is used by a CHA, the device can increase the confidence the caregivers have in the messages being transmitted and increase the confidence the CHAs have in their own work. ${ }^{32} 3337384041$ Through implementation science, innovations in mobile ICTs and strategies for child health and development can be extended to low resource settings to empower local actors and spread the benefits of advancements in technology. ${ }^{3536}$

A study on the performance of CHAs in the Peruvian Amazon identified difficulties they faced in their work. They had difficulty providing a clear explanation of the causes of common diseases and diversifying the health topics that were discussed. ${ }^{29}$ As described above, an mHealth tool could help the CHAs overcome some of the difficulties they have in conducting an effective home visit. However, there is currently little evidence on the impact of an mHealth tool when used as an educational device by CHAs to improve child development. ${ }^{33}$ Additionally, there is a knowledge gap on the impact that an mHealth tool can have when utilised by CHAs in rural regions of Peru. Therefore, the present study aims to demonstrate the impact of an mHealth tool used for health promotion and surveillance by CHAs to improve child development in the Peruvian Amazon.

\section{HYPOTHESIS}

The study hypothesises that an mHealth tool with educational content developed for the local context will improve the capacity of the CHAs to transmit health messages to the caretakers, thus creating behaviour change that will lead to better health and child development outcomes for the children. The hypothesis is based on a theory of change that tracks the mediating variables and considers the potential outputs and outcomes generated by the intervention. ${ }^{42}$ The theory of change displayed in figure 1 highlights the various components that are included in the tablet-based application and unfolds the expected outcomes of each. The theory of change is presented as a comparison to the status-quo; traditional CHA programme in Peru that use paper-based reporting and educational material.

Based on the theory of change, the study has the following hypotheses:

H1: The mHealth tool will improve the performance of CHAs during their home visits with children, as measured by doses, content and interpersonal relationships.

H2: Improved performance by CHAs will increase knowledge acquisition and healthy child-rearing practices by caregivers.

H3: Improved knowledge and healthy practices by caregivers will improve the health and development of the children that receive the intervention.

\section{OBJECTIVES}

\section{Main aim}

The objective of the study is to evaluate the impact of the mHealth tool (tablet with application) on child development and nutrition status of children aged 6 to 36 months compared with children who receive home visits from CHA without the mHealth tool.

\section{Primary outcomes}

1. (Primary Objective) Improve child development scores for the children in the intervention group in the domains of language, cognition, gross motor and socialemotional by $20 \%$ compared with the children in the control group $(\mathrm{p}<0.05)$.

2. Decrease the prevalence of anaemia in children by $20 \%$ compared with the baseline measurement.

a. Children in the intervention group will experience a greater reduction in the prevalence of anaemia than children in the control group $(\mathrm{p}<0.05)$.

3. Decrease the prevalence of chronic malnutrition in children by $15 \%$, compared with the baseline measurement.

a. Children in the intervention group will experience a greater reduction in the prevalence of chronic malnutrition than children in the control group $(\mathrm{p}<0.05)$. 

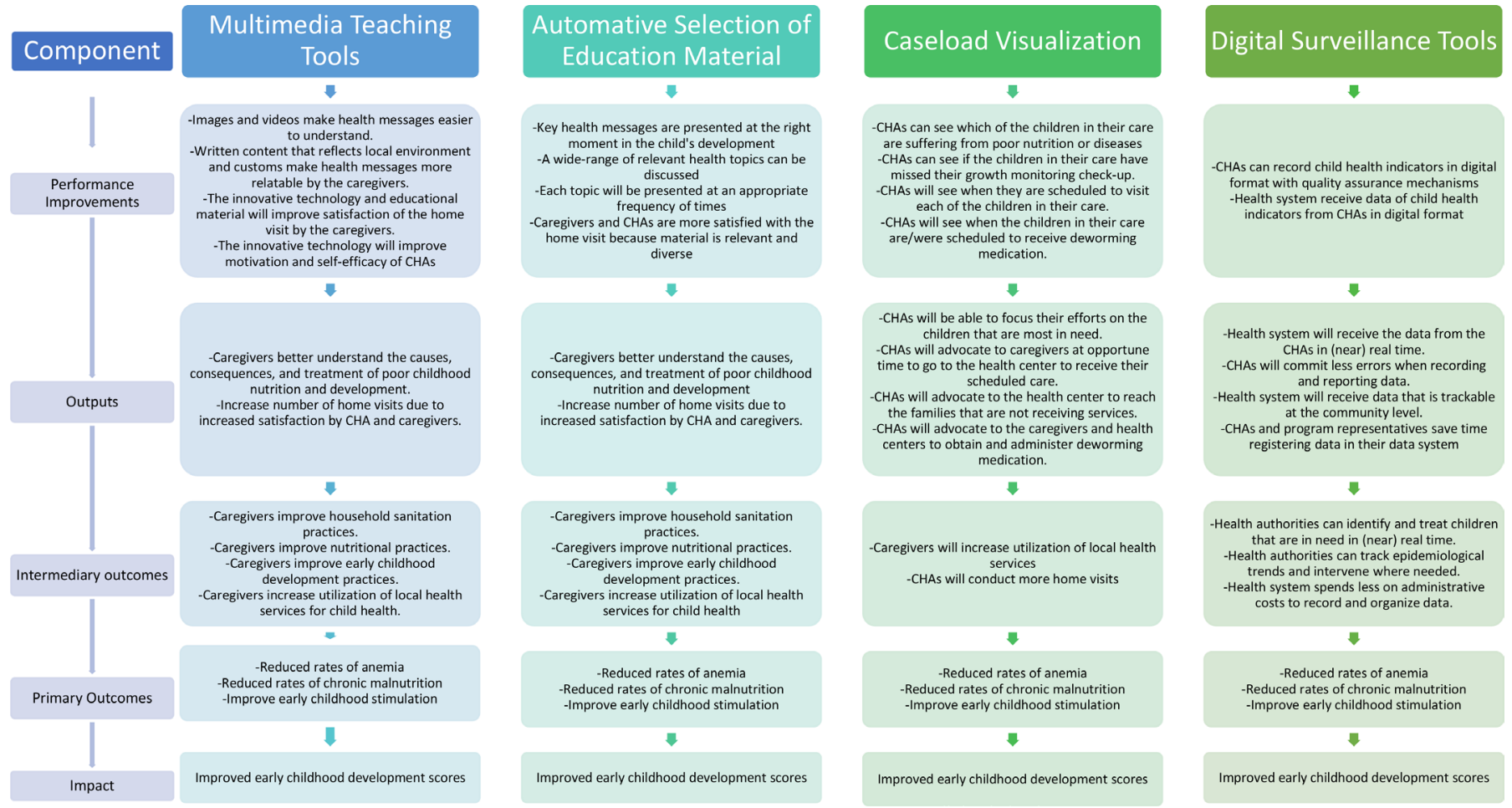

Figure 1 Theory of change. CHA, community health agent.

\section{Secondary outcomes}

The secondary outcomes of the intervention include indicators of behaviour change by CHA and caregivers that have established causal connections with improved health and child development. The intervention is expected to achieve the following secondary outcomes:

4. The caregivers of the intervention group will improve knowledge evaluation scores by $50 \%$ compared with the baseline measurement.

5. CHAs in the intervention group will improve their performance evaluation scores by $50 \%$ compared with the baseline measurement.

6. More than $50 \%$ of the CHAs in the intervention group will express improved satisfaction and self-efficacy when compared with the baseline data.

7. The caregivers of the intervention group will use the local health services for children's health more frequently than they did before baseline.

\section{METHODS}

\section{Study design}

The impact will be evaluated through an experimental cluster randomised controlled trial. Each cluster represents a CHA because the children that receive visits from the same CHA must receive the same intervention. Each cluster will be matched with a second cluster using covariate-constrained randomisation. ${ }^{43}$ Recruitment and baseline surveys will be conducted before allocation to the intervention or control group for each cluster. The variables that will be used to covariate-constrained randomisation include: the size of the community, type of health facility in the community, distance to the nearest health centre (level I-2) and prevalence of anaemia. For each cluster pair, one cluster will be randomly assigned to the intervention group and the other to the control group. The covariate-constrained randomisation will be conducted with an open-sourced algorithm and the program, R. ${ }^{44}$

\section{Location}

The study will be implemented in the Amazon region of Peru. The study will be carried out in the department of Loreto, province of Maynas, and districts of Mazan, Punchana and Indiana. The district of Mazan has a population of $13779,{ }^{45}$ a prevalence of chronic malnutrition of $24.4 \%$ and a prevalence of anaemia at $39.1 \%$, in $2017 .{ }^{46}$ The capital of the district of Mazan is located approximately 1-1/2 hours by boat from the capitol city of the province, Iquitos. The district of Punchana has a population of $91128,{ }^{45}$ a prevalence of chronic malnutrition of $22.7 \%$ and a prevalence of anaemia of $39.9 \% .{ }^{46}$ The capital of Punchana, is $20 \mathrm{~min}$ from the city of Iquitos. The district of Indiana has a population of $11301,{ }^{45} \mathrm{a}$ prevalence of chronic malnutrition of $22.4 \%$ and a prevalence of anaemia at $46.7 \%$, in $2017 .{ }^{46}$ The capital of the district of Indiana is 1.5 hours by boat from the city of Iquitos.

The research team, with the support of the Regional Ministry of Health of Loreto, will choose the communities that will be included in the study after travelling to the communities to ensure they meet the selection criteria. The study communities must have the following characteristics: 
- Has an active CHA that visits families in their community.

- Is not a participant in another health related study.

- Is located less than 6 hours from Iquitos.

- Has at least 25 families and a maximum of 1500 families.

The communities must have an active CHA programme for the study because we are measuring the impact of included the mHealth tool in existing CHA programme. The community cannot be participating in another study that includes an intervention that was/will be implemented due to the confounding variables that would created in our study and the other study. The communities must be less than 6 hours from Iquitos due to budgetary restraints that hinder the research team's ability to consistently travel six or more hours to execute the study. The size limitation of the community is required to ensure the study obtains the minimum number of children to power the study with the limited number of tablets.

\section{Participants and sample size}

The study population includes:

- 10-14 communities, half in the intervention group and half in the control group.

- 40-50 CHAs, half CHAs in the intervention group and half CHAs in the control group.

- 400-450 children, half in the intervention group and half in the control group.

All children between 0 and 36 months in the communities will receive visits from a CHA, in the intervention and control groups. However, only the CHAs in the intervention group will receive a tablet with the application to assist their home visits. All the children in the intervention group will have the opportunity to receive the intervention with the tablet.

To assess the impact of the intervention, a sample of the population will be chosen at random to receive the evaluation. The inclusion criteria for the study sample are the following:

- Children who belong to one of the communities included in the study, aged 6-36 months.

- Primary caregiver of the child is older than 15 years.

- The caregiver is willing to receive visits from the CHA.

The minimum age of the child to be included in the impact evaluation is determined by the quality of the early childhood development tool. Assessment of child development is more accurate with children older than 5 months. ${ }^{47}$ The maximum age of the children for the study is determined by the CHA programme which limits home visits by CHAs to children up to 36 months old. The minimum age of the caregiver is 16 because that is the age determined by the communities and the local ethics committee to be capable of giving an informed consent. It is important to capture information from mothers below 18 years of age because $39 \%$ of the mothers in the region have their first child before the age of $17 .{ }^{4}$ Caregivers who do not want to receive visits from CHAs will be excluded because they will not receive the intervention, nor be included in the control group.

The exclusion criteria for the study sample are:

- Families where the main caregiver or the child does not speak Spanish.

- Families that do not want to receive home visits from CHAs.

- Children with mental or physical disabilities.

Families that do not speak Spanish cannot be included because the study does not have appropriate translators for indigenous languages. Children with disabilities will be excluded in the evaluation because the child development evaluation tool is not equipped to evaluate children with these conditions. However, children with disabilities will still receive the intervention.

The sample size for the evaluation is calculated to measure the effect size on the primary outcome indicator, child development. A study in the Amazon region of Peru (2017) determined that the SD of communication scores, determined by the Ages and Stages Questionnaire, was 15.19. ${ }^{4}$ The current study predicts a $20 \%$ increase in child development scores which will result in an effect size of 0.49 (average communication score was 37.48 , a $20 \%$ increase is $44.98,0.49 \mathrm{SD}$ ) (data available in public repository). ${ }^{4}$ To calculate the minimal sample size needed, the following parameters were determined:

- Two-sided significance level: 0.05 .

- Power: $90 \%$.

- Effect size: 0.49 .

- Average cluster Size: 9.

- Intracluster correlation (ICC): 0.15 .

- Variance inflation factor (VIF): 2.2.

The following calculation is used to determine the sample size:

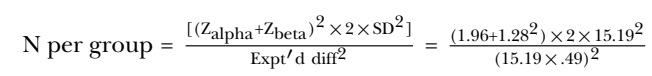

The sample size per group (t-test) with no clusters would be 88 . To control for the ICC, the per group sample size (88) is multiplied by the VIF ( $\mathrm{VIF}=1+$ (average cluster size -1$) * \mathrm{ICC}=2.2$ ) to determine the minimal sample size to be 193 per group. ${ }^{48}$ The study will include 21 clusters, and nine children and caregivers per cluster.

\section{Instruments}

To measure the impact of the intervention and understand the perspective of the users, a series of instruments will be applied to the study participants. The instruments include evaluation of child development, attitudes and practices questionnaire, knowledge evaluation, performance evaluation of CHAs, and collection of anaemia and anthropometric indicators. Qualitative surveys will also be used to evaluate the satisfaction of CHAs and caregivers in relation to the intervention.

The questionnaires and evaluations will be carried out in the homes of the participants. The researcher will ask if a caregiver of a child within the age range is in the home, if so, they will explain the study and ask the caregiver if they want to participant and are willing to sign the informed 
consent. The researcher will record the responses of the questionnaires and evaluation on a smart phone or electronic tablet.

\section{Evaluation of child development}

Child development can be effectively evaluated with an instrument that utilises caregiver-reported data to assess the ability of the child to perform age-relevant developmental activities. ${ }^{47-52}$ The current study will use the Caregiver-Reported Early Development Instrument (CREDI) to evaluate child development. ${ }^{534}$ The CREDI has been validated as an effective instrument to evaluate child development, including low-resource settings in 17 countries. ${ }^{54}$ The CREDI is effective for large-scale, population-wide studies because it requires less time, materials and evaluator expertise than other similar instruments. ${ }^{53}$

The CREDI is conducted by asking the caregiver if the child is currently capable of completing a list of ageappropriate activities, in which the caregiver can respond by indicating; yes, no or I don't know. The CREDI will provide a developmental score and a classification that represents the child's development trajectory. These classifications will be used as the primary dependent variables of the study.

\section{Attitudes and Practices Questionnaire}

The study will use a quantitative questionnaire to measure the attitudes and practices of caregivers that influence maternal and child health. The following topics are included in the questionnaire: demographic information, safe pregnancy practices, feeding practices, prevention of illnesses, early childhood stimulation and use of local health services. The questionnaire will be conducted with all caregivers of the children included in the study. The questionnaire will take approximately $10 \mathrm{~min}$.

\section{Knowledge evaluation}

To assess the knowledge of healthy child-rearing practices by the caregiver, an instrument was created based on the literature. ${ }^{135-57}$ The knowledge evaluation asks the caregiver questions regarding maternal and child health, nutrition, sanitation and infectious diseases. The questions are open-ended to give the caregiver the opportunity to provide multiple answers that display their knowledge of the topic.

The knowledge evaluation will take place directly after the Attitudes and Practices Questionnaire. The knowledge evaluation will be scored by giving the participant one point for every correct answer they provide. The correct answers will be added together to provide a total 'Knowledge Score' for the caregiver.

\section{Performance evaluation of $\mathrm{CHAs}$}

The performance evaluation of CHAs was based on an instrument built by the University of West Indies and the Inter-American Development Bank to measure the quality of home visits by CHAs. ${ }^{14}$ The performance evaluation includes questions related to the use of materials, activities, promotion of child development, relationship between CHA and caregivers, and relationships between the CHA and the child. The performance evaluation was modified and validated during a previous study in the Amazon region within the local CHA programme. The instrument provides further focus on health messages provided by the CHA and the needs of the communities in the Amazon. ${ }^{29}$

To conduct the performance evaluation, the researcher will accompany the CHA on their home visit with a child to observe the home visit. The researcher will observe the home visit in silence, trying not to influence the dynamic of the visit. The researcher will take notes during the visit and complete the performance evaluation immediately after the conclusion of the home visit.

\section{Survey of the use of local health services}

The research team will survey the local health centres in the communities to obtain statistics regarding the use of health services related to maternal and child health, including child growth monitoring check-ups, prenatal check-ups, immunisation coverage, distribution of micronutrient supplements, distribution of antiparasitic medicine and distribution of oral rehydration salts for diarrhoea. The survey will be conducted with the nurse that conducts maternal and child health evaluations at the health centre. The survey will take approximately $15 \mathrm{~min}$ and can be filled out independently by the health post representative.

\section{Survey of child health indicators}

The Child Health Education and Surveillance Tool (CHEST) application will be used to collect child health indicators by CHAs during their home visits. The children have a card with information that was recorded during their growth monitoring check-ups at the local health centre, including height, weight, age, vaccines and haemoglobin levels (when tested). The CHA will observe the information written on the card and record it with the application. The CHA will also ask additional questions regarding the health of the child, including incidences of diarrhoea, cough, fever, malaria and other illness. The data from the application will be uploaded to the secured server when the CHA or supervisor has access to the cellular network.

\section{Questionnaire on Satisfaction and Self-Efficacy of CHAs}

The Questionnaire on Satisfaction and Self-Efficacy of CHAs will be used to measure the degree of satisfaction and self-efficacy the CHAs have from using the CHEST application during their home visits. The questions are both quantitative and qualitative. The CHAs will have the opportunity to describe how the experience with the tablet could be improved.

\section{Questionnaire on Satisfaction of Caregiver}

The Questionnaire on Satisfaction of Caregivers will be used to measure the degree of satisfaction of the caregivers that received the intervention. The questions are 


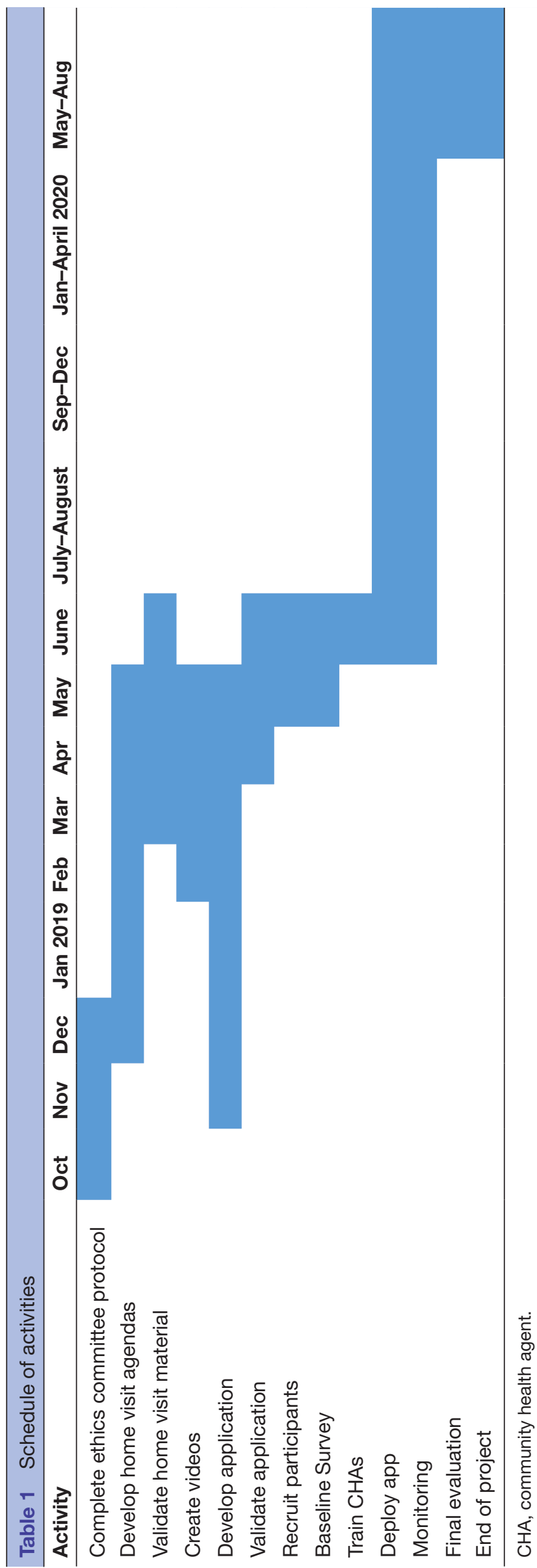

both quantitative and qualitative. The caregivers will have the opportunity to describe what they like and don't like about the use of a tablet during the home visits.

\section{Patient and public involvement}

The study was created through formative research done in the same communities to identify their needs and desires related to improving child health and development. A performance evaluation study was conducted with the CHAs in the region by the author. ${ }^{29}$ In the study, the CHAs and caregivers expressed their need and desire for more information regarding topics of health and child development. The current intervention was created to address those needs.

The results of the study will be presented to the participants, including the CHAs, caregivers, health facility personal and Regional Ministry of Health in the form of a study brief that indicates the results of each indicator collected. The results will be presented to the public via publication of results in an international scientific journal.

Patients were not involved in the design or recruitment of the study.

\section{INTERVENTION}

The intervention includes the development and implementation of a tablet-based application (CHEST) to improve the performance of the CHAs during their home visits. The CHAs will use the CHEST application during their home visits to teach caregivers good health practices and early childhood stimulation. The application will provide the steps for the CHA to follow to conduct an effective home visit. The application will include videos and images to show the caregiver to transmit key health messages. Also, the application will organise the case load of children of the CHA and will provide a mechanism to record maternal and child health indicators.

The intervention is described in the following five phases:

\section{Phase 1: preparation of material}

\section{Step-by-step agendas for home visits}

The research team will work with the Regional Ministry of Health of Loreto to build agendas for home visits by the CHAs. The health messages for the home visits are based on national and international manuals for home visits by CHAs to teach maternal and child health and development. ${ }^{58-61}$ The agendas include steps to follow by the CHA to provide a quality home visit and collect child health indicators. The messages included in the agendas include; what nutritious foods should be consumed by children, how to avoid diarrhoea and other diseases, good practices of early attention and use of health services. There will be one agenda for each month of age of the child (1-36 months).

\section{Multimedia material}

The research team will generate multimedia material to be used in the application to transmit health messages. The 


\begin{tabular}{|c|c|c|c|c|}
\hline \multirow[b]{3}{*}{ Timepoint } & \multicolumn{4}{|c|}{ Study period } \\
\hline & \multirow{2}{*}{$\begin{array}{l}\text { Enrolment } \\
-t_{1}\end{array}$} & \multirow{2}{*}{$\begin{array}{l}\text { Allocation } \\
0 \\
\end{array}$} & Post-allocation & \multirow{2}{*}{$\begin{array}{l}\text { Close-out } \\
t_{\mathrm{x}}\end{array}$} \\
\hline & & & $t_{2}$ & \\
\hline \multicolumn{5}{|l|}{ Enrolment: } \\
\hline Eligibility screen of inclusion and exclusion criteria & $x$ & & & \\
\hline Informed consent & $x$ & & & \\
\hline Allocation & & $x$ & & \\
\hline \multicolumn{5}{|l|}{ Interventions } \\
\hline Distribution of tablet & & & $\longleftrightarrow$ & \\
\hline Control group & & & $\rightleftarrows$ & \\
\hline \multicolumn{5}{|l|}{ Assessments } \\
\hline Child development evaluation & $x$ & & & $x$ \\
\hline Performance evaluation & $x$ & & & $x$ \\
\hline Knowledge, Attitudes and Practices Survey & $x$ & & & $x$ \\
\hline Monitoring of community health agents & & $\mathrm{x}$ & $x$ & \\
\hline
\end{tabular}

content will be designed to convey the health messages clearly within the local culture in the Amazon. The material will include videos of people from the region acting out health topics, instructional videos on early childhood stimulation and animations that describes health issues. In addition, the application will include educational images that have been developed by the regional Ministry of Health of Loreto and other institutions.

\section{Phase 2: development of the application}

The research team will evaluate the mHealth applications that have been developed and are open source to determine which will be best to adapt for the current project. The project will contract a team of programme developers to modify the open source application to fit the current objectives and local context. The programme developer will include the agendas, multimedia and data collection mechanisms in the application. The application will function without internet signal and protect all information by password.

\section{Phase 3: baseline data collection}

The research team will conduct the surveys and evaluations in the intervention and control communities to obtain a baseline for the study. The instruments that will be applied for the baseline survey include the Child Development Evaluation, Attitudes and Practices Questionnaire, Knowledge Evaluation, Performance Evaluation of CHA and Survey on the Use of Health Services. The baseline survey will be completed before implementation begins in the intervention and control communities.

\section{Phase 4: implementation of intervention}

Training workshops

The research team will conduct training workshops with the CHAs in the intervention group to teach them how to use the tablet and application. The workshops will occur during a period of 3 days, 4 hours per day. The leaders of the health facility and Regional Ministry of Health of Loreto will be invited to participate in the workshops and in the supervision of the development of the project.

\section{Distribution of tablets}

After the training workshops, the research team will give a tablet to each CHA in the intervention communities. The CHAs will be instructed to bring the tablet to local health centre if they are having problems with the tablet or to store the tablet in their home until a member of the monitoring team visits their community. A supervisor of the CHA programme will visit the CHAs to ensure the app is being used and functioning correctly.

\section{Intervention}

The CHAs will continue to conduct home visits with the children in their caseload the same as before the distribution of the tablets. The only change is that the CHAs will use the tablets during the home visits. The number of home visits depends on the demands placed by the community and the motivation of the individual CHA. The CHAs in the communities typically conduct 1-4 home visits per month with children aged 0-36 months. The CHAs are instructed to teach the caregivers good sanitation, nutrition and child development practices.

The CHAs will use the tablet-based app to visualise the health indicators of the children in their caseload, record health indicators of the child during each visit, observe and share images and videos that will help teach health and development messaging, and send data to a central database. The app will guide the CHA through each step of the home visit. The supervisor of the CHA programme will visit the CHAs to ensure they can upload their data to the database. This include, in some cases, carrying the tablet to a community that has signal to upload the data. 


\section{Monitoring}

The research team will supervise the work of the CHAs through observations of their home visits and interviews with families and CHAs in the communities. The research team will visit the communities every month during the first 4 months, and less frequently when they see that the programme is working as it should.

The team will also track activities of the CHAs through the data uploaded by the application. They will be able to track the number of visits being conducted and the health indicators of the children. If the tablet is not uploading data to the survey, the research team will visit the community to identify the problem.

The local health facility will also have access to the data and will be able to identify if a child is at risk of chronic malnutrition, chronic diarrhoea and/or is not attending growth monitoring check-ups.

\section{Phase 5: evaluation of the intervention}

At the end of the intervention, the follow-up survey will be carried out in the intervention and control communities. The instruments included in the follow-up survey include the Evaluation of Child Development, Attitudes and Practices Questionnaire, Knowledge Test, Performance Evaluation of CHA, Survey of Use of Health Services, Questionnaire on Satisfaction and Self-Efficacy of CHA and Questionnaire on Satisfaction of Caregivers.

The data gathered by the application will be used to evaluate the child heath indicators and the number of home visits conducted.

\section{DATA ANALYSIS}

The quantitative data obtained in the surveys will be organised and analysed with the statistical software, Epi Info V.7.1.4.0. ${ }^{62}$ The qualitative data will be organised and analysed with the program Atlas.ti. ${ }^{63}$ To carry out the impact analysis, four mathematical models will be used to interpret the results, generating an optimal analysis to understand the interactions between the variables. The analysis will be conducted on the dependent variable to test the primary outcome, as well as the intermediary variables to test the secondary outcomes and measure the mediating effect of those variables. First, a descriptive analysis will be performed to compare indicators between the analysis groups, including average and frequencies of the independent and dependent variables. Second, a bivariate analysis with $\chi^{2}$ test will be done to examine the associations between the independent and the dependent variables. Third, a multivariate regression analysis will be used to examine the interactions between the independent variables and the dependent variables. Fourth, an analysis of covariance regression method will be used to calculate the impact of the intervention. ${ }^{43}$

\section{Data monitoring}

Data monitoring will be completed by the data monitoring committee to ensure non-biased handling and analysis of data obtained from baseline, monitoring and follow-survey. The data monitoring committee will be made up of the team of experts that will be hired to create the content of the intervention and mentors that guided the research team. The data monitoring committee will each guard files of data at different time points so they can later be cross-referenced to ensure consistency. The data monitoring committee will not include permanent members of the sponsor organisation or associates of the funding organisation or anyone with competing interest or incentive for the intervention to be successful.

\section{Data security}

All data will be stored in a secure encrypted server that is protected by password. All identifiable information in the data set will be deleted to make it impossible to link the data with the individual. Data obtained during follow-up does not need to be connected with individual cases from the baseline, therefore, identifying information does not need to be stored. Information regarding contact information of CHAs will be stored on a secure server. Members of the data monitoring committee will ensure all identifiable information has been removed from the data set.

\section{ETHICS AND DISSEMINATION}

The investigation will include only those individuals who are willing and able to give their consent to be included in the study. The participant must be able to understand the information presented to them and voluntarily express their choice of whether they want to participate or not. The caregivers must be over 15 years of age to participate in the study.

It will be clearly stated that participation in the study will not compromise the health of the participants or violate any rights of the child. There will be no financial compensation for participation in the study. No biological samples will be collected, nor any medical or pharmacological treatments delivered in the research process. There are no possible adverse effects associated with participation in the study. There is the possibility of generating discomfort in the participants because the visits and surveys imply an investment of time. Given this, participants will be informed about the amount of time involved for their participation in the survey before signing the informed consent.

After completion of the child-development evaluation, the results will be immediately shared with the caretaker along with recommendations of early childhood stimulation activities that could benefit the child. Likewise, the results of the evaluations will be communicated to the health centre of the community with a copy of a list of the children who are at risk of developmental delay. The child development evaluation represents a direct benefit to the participants in the intervention and control groups.

The research team will monitor the intervention for at least 12 months to ensure the CHAs receive support, and 
the tablets receive maintenance or are replaced if need. The CHAs can opt out of the intervention at any time, thus returning to their traditional methods of reporting and health promotion. After 12 months, the programme will be handed over to the municipality and they will continue to receive support as needed. The municipality has been active in the design of the material for the intervention and will be active in the monitoring and evaluation efforts. The municipality members will be trained in how to manage and sustain the programme.

The results obtained from the study will be made publicly accessible through the publication of findings in an international peer-reviewed journal and a report will be delivered to the local health authorities. The dataset will available in a public data repository.

\section{Informed consent for caregivers}

The informed consent for caregivers includes the description of the study, the participation of the caregiver, the participation of the child and the amount of time required to complete the survey. The interviewer that conducts the questionnaires will ask for consent from the caregiver. If the caregiver agrees to participate in the study, they will be asked to sign the informed consent form. The caregiver will be provided a copy of the consent.

\section{Informed consent for CHA}

The informed consent for CHAs includes a description of the study and the performance evaluation that will be conducted with the CHA. The interviewer who conducts the questionnaires will ask for consent from the CHA. If the CHA agrees to participate in the study, they will be asked to sign the informed consent for CHAs. The CHA will be offered a copy of the consent.

\section{Consent to publish}

There will be no identifying images, personal details or clinical details of participants presented that will compromise the anonymity of the participants in the study.

\section{CONCLUSIONS}

The results of the study will provide evidence on the potential of a mHealth tool to improve child health and development indicators in the region. The process evaluation with qualitative assessments will also be conducted to identify barriers, strengths and weaknesses of the programme.

\section{CHRONOLOGY}

The study will last 24 months, beginning in October 2018 and ending in September 2020. The schedule of activities can be found in table 1 .

\section{Flow diagram schedule of activities}

The flow diagram in table 2 describes when intervention allocation and assessments of the study population will take place, in relation to the study activities.

\section{Communication of trial results}

The results of the study will be presented to the participants, including the CHAs, caregivers, health facility personal and Regional Ministry of Health in the form of a study brief that indicates the results of each indicator collected. The study brief will be written and described in a manner that is intelligible for the target population. The CHAs will be asked to distribute the brief and describe the findings to the individual families in their caseload. The results will be presented to the public via publication of results in an international scientific journal. The research team will also seek opportunities to present the results in national and international conferences to disseminate findings.

Acknowledgements Special thanks to Liz Franco, Luis Orrego Ferreyros, Milagros Alvarado Llatance and Gabriela Palacios Rojo for their assistance in developing the material and instruments of the study. Thanks to the Ministry of Development and Social Inclusion of Peru for their collaboration in designing the tool and identifying the participants and recipients of the study. Special thanks to Grand Challenges Canada for providing advisory service regarding study design, implementation strategies and strategies to scale the intervention.

Contributors CW conceived of the study. CW and NR contributed to the writing and revision of the protocol. PM provided advisory on study design. All authors contributed to refinement of the study protocol and approved the final manuscript.

Funding The study is funded by the grant, Saving Brains, by Grand Challenges, Canada.

Disclaimer The funding source had no role in the design of this study and will not have any role during its execution, analyses, interpretation of the data or decision to submit results.

Competing interests None declared.

Patient consent for publication Not required.

Ethics approval The investigation was approved by the Institutional Ethics Committee of the National Hospital 'San Bartolomé' in Peru on November 8, 2018 (Exp. Number 15 463-18, Oficio N. 0744-2018-0ADI-HONADOMANI-SB).

Provenance and peer review Not commissioned; externally peer reviewed.

Data availability statement There are no data in this work. All data relevant to the study are included in the article or uploaded as supplementary information.

Open access This is an open access article distributed in accordance with the Creative Commons Attribution Non Commercial (CC BY-NC 4.0) license, which permits others to distribute, remix, adapt, build upon this work non-commercially, and license their derivative works on different terms, provided the original work is properly cited, appropriate credit is given, any changes made indicated, and the use is non-commercial. See: http://creativecommons.org/licenses/by-nc/4.0/.

ORCID iD

Christopher Michael Westgard http://orcid.org/0000-0002-8914-4021

\section{REFERENCES}

1 Grantham-McGregor S, Cheung YB, Cueto S, et al. Developmental potential in the first 5 years for children in developing countries. Lancet 2007;369:60-70.

2 Engle PL, Black MM, Behrman JR, et al. Strategies to avoid the loss of developmental potential in more than 200 million children in the developing world. Lancet 2007;369:229-42.

3 Walker SP, Wachs TD, Grantham-McGregor S, et al. Inequality in early childhood: risk and protective factors for early child development. Lancet 2011;378:1325-38.

4 Westgard C, Alnasser Y. Developmental delay in the Amazon: the social determinants and prevalence among rural communities in Peru. PLoS One 2017;12:e0186263.

5 Instituto Nacional de Estadistica E Informatica. Loreto Compendio Estadistico 2017. Sistema Estadistico Nacional 2017. 
6 Anticona C, San Sebastian M. Anemia and malnutrition in Indigenous children and adolescents of the Peruvian Amazon in a context of lead exposure: a cross-sectional study. Glob Health Action 2014;7:22888.

7 Díaz A, Arana A, Vargas-Machuca R, et al. Situación de salud y nutrición de niños indígenas y niños no indígenas de la Amazonia peruana. US11 [Internet], 2015. Available: http://iris.paho.org/xmlui/ handle/123456789/10008 [cited 2017 Jan 26].

8 Flores-Bendezu J, Calderon J, Rojas B, et al. Chronic malnutrition and anemia in children under 5 years of indigenous households of Peru - Analysis of Demographic and Health Survey 2013. Fac Med 2015;76:2135-40.

9 Grantham-McGregor SM, Fernald LCH, Kagawa RMC, et al. Effects of integrated child development and nutrition interventions on child development and nutritional status. Ann N Y Acad Sci 2014:1308:11-32.

10 Perry HB, Sacks E, Schleiff M, et al. Comprehensive review of the evidence regarding the effectiveness of community-based primary health care in improving maternal, neonatal and child health: 6 . strategies used by effective projects. $J$ Glob Health 2017:7:010906.

11 Gilmore B, McAuliffe E. Effectiveness of community health workers delivering preventive interventions for maternal and child health in low- and middle-income countries: a systematic review. BMC Public Health 2013;13:847.

12 Global Health Workforce Alliance, World Health Organization. Global experience of community health workers for delivery of health related millennium development goals: a systematic review, country case studies, and recommendations for integration into National health systems 2010.

13 The Earth Institute. Millennium Villages Project. Community Health Worker Trainer's Manual. A guide to Home Based Services [Internet]. Version 2.1. Columbia University, 2013. Available: http://millenniumvillages.org/wp-content/uploads/2014/09/ CHWTrainingManual.pdf

14 Leer J, Lopez-Boo F, Perez Exposito A, et al. A snapshot on the quality of seven home visit parenting programs in Latin America and the Caribbean. Inter-American development bank 2016.

15 Schodt S, Parr J, Maria CA, et al. La medición de la calidad de os servicios de visitas domiciliarias. Una revisión de la literatura. Danco Interamericano de Desarrollo 2015.

16 Vogel CA, Caronongan P, Thomas J. Toddlers in Early Head Start: A Portrait of 2-Year-Olds, Their Families, and the Programs Serving Them. Volume 1: Age 2 Report. OPRE Report 2015-10 [Internet]. Administration for Children \& Families, 2015. Available: https://eric. ed.gov/?id=ED558558 [cited 2017 Aug 3].

17 Roggman LA, Cook GA, Innocenti MS, et al. Home visit quality variations in two early head start programs in relation to parenting and child vocabulary outcomes. Infant Ment Health $J$ 2016;37:193-207.

18 Medina A, Sofía I. Evaluation protocol application of tasks for the socio - cognitive development in children from 37 to 71 months in the Program of Comprehensive Attention to Early Childhood Modality Family Environment District Secretariat of Social Integration [Internet]. Available: https://intellectum.unisabana.edu.co/handle/ 10818/23587 [cited 2017 Aug 3].

19 Fernández S, Yonah R. Aplicación de ensayos domiciliarios para evaluar la adopción de prácticas mejoradas de alimentación complementaria en madres. Centro materno infantil Juan Pablo II. Villa El Salvador, Lima. 2005. Univ Nac Mayor San Marcos [Internet], 2007. Available: http://cybertesis.unmsm.edu.pe/handle/cybertesis/ 1121 [cited 2017 Aug 4].

20 Walker SP, Powell C, Chang SM, et al. Estrategias de apoyo parental a través de los servicios de salud en el Caribe [Internet]. BID, 2016. Available: http://repositorio.minedu.gob.pe/handle/123456789/4112 [cited 2017 Aug 3].

21 Chang-Say Chong A. La comunicación en los proyectos de lucha contra la desnutrición crónica infantil : el caso Ally Micuy (Áncash). Pontif Univ Católica Perú [Internet], 2014. Available: http://tesis.pucp. edu.pe/repositorio/handle/123456789/5598 [cited 2017 Aug 3].

22 Lewin S, Munabi-Babigumira S, Glenton C, et al. Lay health workers in primary and community health care for maternal and child health and the management of infectious diseases. Cochrane Database Syst Rev 2010;94.

23 Lassi ZS, Kumar R, Bhutta ZA. Community-Based Care to Improve Maternal, Newborn, and Child Health. In: Black RE, Laxminarayan R, Temmerman M, eds. Reproductive, Maternal, Newborn, and Child Health: Disease Control Priorities, Third Edition (Volume 2) [Internet]. Washington (DC): The International Bank for Reconstruction and Development / The World Bank, 2016.

24 Howard KS, Brooks-Gunn J. The role of home-visiting programs in preventing child abuse and neglect. Future Child 2009;19:119-46.
25 Singh V, Ahmed S, Dreyfuss ML, et al. Non-governmental organization facilitation of a community-based nutrition and health program: effect on program exposure and associated infant feeding practices in rural India. PLoS One 2017:12:e0183316.

26 Swider SM. Outcome effectiveness of community health workers: an integrative literature review. Public Health Nurs 2002;19:11-20.

27 Agarwal S, Perry HB, Long L-A, et al. Evidence on feasibility and effective use of mHealth strategies by frontline health workers in developing countries: systematic review. Trop Med Int Health 2015;20:1003-14.

28 Mobile Healthcare Information for All. Assessment of mHealth applicaitons for their potential to provide essential healthcare information for citizens in low resource settings. HIFA 2017.

29 Westgard C, Naraine R, Paucar Villacorta DM. Performance evaluation of community health workers: case study in the Amazon of Peru. J Community Health 2018.

30 Ballard M, Montgomery P. Systematic review of interventions for improving the performance of community health workers in lowincome and middle-income countries. BMJ Open 2017;7:e014216.

31 Cometto G, Ford N, Pfaffman-Zambruni J, et al. Health policy and system support to optimise community health worker programmes: an abridged who guideline. Lancet Glob Health 2018;6:e1397-404.

32 llozumba O, Dieleman M, Kraamwinkel N, et al. "I am not telling. The mobile is telling": Factors influencing the outcomes of a community health worker mHealth intervention in India. PLoS One 2018;13:e0194927.

33 van Heerden A, Sen D, Desmond C, et al. App-supported promotion of child growth and development by community health workers in Kenya: feasibility and acceptability study. JMIR mHealth and uHealth 2017;5.

34 Lee S, Chib A, Kim J-N. Midwives' cell phone use and health knowledge in rural communities. $J$ Health Commun 2011;16:1006-23.

35 Peterson HB, Haidar J, Fixsen D, et al. Implementing innovations in global women's, children's, and adolescents' health: Realizing the potential for implementation science. Obstet Gynecol 2018;131:423-30.

36 Peterson HB, Haidar J, Merialdi M, et al. Preventing maternal and newborn deaths globally: using innovation and science to address challenges in implementing life-saving interventions. Obstet Gynecol 2012;120:636-42.

37 Källander K, Tibenderana JK, Akpogheneta OJ, et al. Mobile health (mHealth) approaches and lessons for increased performance and retention of community health workers in low- and middle-income countries: a review. J Med Internet Res 2013;15:e17.

38 Prinja S, Nimesh R, Gupta A, et al. Impact of m-health application used by community health volunteers on improving utilisation of maternal, new-born and child health care services in a rural area of Uttar Pradesh, India. Trop Med Int Health 2017:22:895-907.

39 Ilozumba O, Van Belle S, Dieleman M, et al. The effect of a community health worker utilized mobile health application on maternal health knowledge and behavior: a quasi-experimental study. Front. Public Health 2018;6.

40 Chib A. The Aceh Besar midwives with mobile phones project: design and evaluation perspectives using the information and communication technologies for healthcare development model. $J$ Comput-Mediat Commun 2010;15:500-25.

41 Limaye NP, Rivas-Nieto AC, Carcamo CP, et al. Nuestras Historiasdesigning a novel digital story intervention through participatory methods to improve maternal and child health in the Peruvian Amazon. PLoS One 2018;13:e0205673.

42 Lafferty CK, Mahoney CA. A framework for evaluating comprehensive community initiatives. Health Promot Pract 2003;4:31-44.

43 Ivers NM, Halperin IJ, Barnsley J, et al. Allocation techniques for balance at baseline in cluster randomized trials: a methodological review. Trials 2012;13:120.

44 Carter BR, Hood K. Balance algorithm for cluster randomized trials. BMC Med Res Methodol 2008;8:65.

45 Instituto Nacional de Estadistica E Informatica. Estadisticas. Poblacion y Vivienda. Poblacion Ttoal al 30 de Junio, POr Grupos Quinquenales de Edad, Segun Departamento, Provincia y Distrito, 2015 [Internet], 2015. Available: https://www.inei.gob.pe/ estadisticas/indice-tematico/poblacion-y-vivienda/

46 Instituto Naciona De Salud. Sistema de informacion del estado nutricional (SIEN), 2017. Available: https://web.ins.gob.pe/es/ alimentacion-y-nutricion/vigilancia-alimentaria-y-nutricional/ vigilancia-del-sistema-de-informacion-del-estado-nutricional-en-\% 20EESS

47 Rubio-Codina M, Araujo MC, Attanasio O, et al. Concurrent validity and feasibility of short tests currently used to measure 
early childhood development in large scale studies. PLoS One 2016;11:e0160962.

48 Kelsey J, Whittemore A, Evans A, et al. Methods in Observational Epidemiology. In: Monographs in epidemiology and biostatistics. Vol. 26. 2nd edn. New York: Oxford University Press, 1996.

49 Fernald LCH, Kariger P, Hidrobo M, et al. Socioeconomic gradients in child development in very young children: evidence from India, Indonesia, Peru, and Senegal. Proc Natl Acad Sci U S A 2012;109:17273-80.

50 Fernald L, Kariger P, Engle P, et al. Examining early child development in low-income countries: a toolkit for the assessment of children in the first five years of life. The World Bank, 2009.

51 Rubio-Codina M, Araujo MC, Attanasio O, et al. Validez concurrente y viabilidad de pruebas cortas comunmente usadas para medir el desarrollo infantil temprano en estudios a gran escala: Metodologia y resultados. Banco Interam Desarro [Internet], 2016. Agosto;(723). Available: https://publications.iadb.org/bitstream/handle/11319/ 7823/Validez-concurrente-y-viabilidad-de-pruebas-cortascomunmente-usadas-para-medir-el-desarrollo-infantil-temprano-enestudios-a-gran-escala-metodologia-y-resultados.pdf

52 Rubio-Codina M, Attanasio O, Grantham-McGregor S. Mediating pathways in the socio-economic gradient of child development: evidence from children 6-42 months in Bogota. Int J Behav Dev 2016;40:483-91.

53 McCoy DC, Waldman M, Fink G. Measuring early childhood development at a global scale: evidence from the CaregiverReported early development instruments. Early Child Res $Q$ 2018;45:58-68

54 McCoy DC, Fink G. CREDI. Caregiver-Reported Early DevelopmentInstruments. User Guide. [Internet], 2018. Available: sites.sph.harvard.edu/credi/

55 Tran NT, Portela A, de Bernis L, et al. Developing capacities of community health workers in sexual and reproductive, maternal, newborn, child, and adolescent health: a mapping and review of training resources. PLoS One 2014;9:e94948.

56 Crigler L, Hill K, Furth R. Community health worker assessment and improvement matrix (CHW AIM): a toolkit for improving community health worker programs and services. Revised version. Published by the USAID health care improvement project. Bethesda, MD: University Research Co., LLC, 2013.

57 Basic support for Institutionalizing child survival (basics) and the prevention of postpartum, hemorrhage initiative (POPPHI). training community health workers to give maternal and newborn health messages: a guide for trainers. Arlington, Va., USA: for the United States Agency for International Development 2009.

58 Perry H, Crigler L. Developing and Strengthening Community Health Worker Programs at Scale: a reference guide and case studies for program managers and policymakers. [Internet]. Maternal and Child Health Integrated Program, 2014. Available: http://www.mchip.net/ CHWReferenceGuide

59 Ministerio de Salud. Preparando al Agente COmunitario de Salud para el Cuidado Integral dela Salud y Nutricion de las Gestantes y de las Niñas y Niños Menores de 5 años - "El Manualdel Agente Comunitario de Salud." Ministerio de Salud del Peru 2009.

60 Ministerio de Salud dG de P de la S. Orientaciones para El Fortalecimiento de la labor del Agente Comunitario de Salud. Ministerio de Salud del Peru 2014.

61 Organizacion Panamerican de la Salud. Manual del Agente Comunitario de Salud: manual del Facilitador. Organizacion Mundial de la Salud 2010.

62 Epi InfoTM. CDC [Internet], 2017. Available: https://www.cdc.gov/ epiinfo/index.html [cited 2018 Sep 13].

63 ATLAS.ti: The Qualitative Data Analysis \& Research Software [Internet]. Available: https://atlasti.com/ [cited 2018 Sep 13]. 